(C) 2012 IEEE. Personal use of this material is permitted. Permission from IEEE must be obtained for all other uses, in any current or future media, including reprinting/republishing this material for advertising or promotional purposes, creating new collective works, for resale or redistribution to servers or lists, or reuse of any copyrighted component of this work in other works. 


\title{
Image Processing for Pathological Visualization in Multitemporal Convoluted TIRI
}

\author{
Daniel TJ. Arthur, Masood M. Khan, and Luke C. Barclay
}

\begin{abstract}
The convoluted nature of thermal infrared radiation and poor understanding of the physical mechanisms of human emittance, make objective image acquisition and processing protocols prerequisite for meaningful diagnostic specificity. A longitudinal dataset of clinical thermal infrared images was objectively processed to facilitate visualization of osseous stress pathology in the lower limbs.. This paper details processing of 500+ thermal infrared images acquired during a recent three month clinical study into osseous stress pathology in the lower limbs of Australian Army basic trainees. The use of thermal chroma-keying in segmentation and multitemporal image calibration is demonstrated. The 'OpenSURF' implementation of the scale and rotation-invariant interest point detector and descriptor are shown to be performant in registration of multitemporal clinical thermal infrared image data. Thermal 'signs' observed in longitudinal images appear to be revealing detectable changes in osseous stress pathophysiology.
\end{abstract}

Index Terms - Thermal infrared imaging, medical image processing, thermal image calibration, osseous stress pathology.

\section{Motivation}

$\mathrm{I}$ $\mathrm{N}$, conventional biometric applications, thermal infrared imaging (TIRI) constitutes one of a multispectral suite of remote sensing modalities tasked with bimodal classification $[1,2]$. In a clinical or laboratory setting however, one may consider more demanding and potentially more revealing modes of application [3]. Firstly, TIRI hardware may be readily configured to give sufficient spatial resolution at the region of interest (ROI) for meaningful pixel-based analyses, and secondly, laboratory conditions allow for quantitative deconvolution of measured emittance via mapping of spectral emissivity [4]. Such experimentally derived anatomic emissivity maps (e-maps) may reveal inter-subject and/or broad spectrum consistencies upon which precise quantitative thermography of human ROI's may be based. During a recent three month clinical pilot study into TIRI-based detection of osseous stress pathology in the lower legs of Australian Army basic trainees [5], a dataset of over 500 TIRI's was amassed, with MRI and nuclear scintigraphic validation of clinical fracture diagnosis. This paper describes the selection, optimization, and application of a series of image processing techniques to this dataset, with a view to facilitation of objective TIRIbased visualization of osseous stress pathology.

\section{PRe-Processing}

When physical bodies exhibit temperatures within the range characteristic to in-vivo human tissues, emittance becomes suprathreshold at wavelengths greater than $3 \mu \mathrm{m} \mathrm{[4}$, 6]. In passive broadband TIRI (as opposed to interferometric / spectroscopic modes [7]) this emittance is read out as a convoluted function of both the temperature and emissivity of the subjective body, where emissivity $(\epsilon)$ is a coefficient describing radiative properties as a ratio of said spectral emittance to that from a notional ideal 'blackbody' emitter of the same temperature, as in (1);

$$
W_{\lambda T \psi}=\varepsilon_{\lambda T \psi} \frac{2 \pi h c^{2}}{\lambda^{5}\left(e^{\frac{h c}{\lambda k T}}-1\right)}\left[W \cdot m^{-2} \mu m^{-1}\right]
$$

Where; $\lambda=$ wavelength of subjective emittance, $\mathrm{T}=$ temperature of the physical body, $\psi=$ emission angle, $\mathrm{C}=$ emissivity of the subjective physical body, $\mathrm{h}=$ Planck's constant, $\mathrm{c}=$ the speed of light, and $\mathrm{k}=$ Boltzmann's constant. The pixel graylevels in the subjective database map directly to this emittance, with the steps required to process longitudinal clinical data being analogous to those multitemporal remote sensing [8], yet afforded greater controls during image acquisition [4].

\section{A. Segmentation}

As detailed in the image acquisition protocols of [5], a backing mat of uniform thermo-optical properties was placed directly beyond the ROI to facilitate subsequent image segmentation. Segmentation of the human ROI from the background is conducive to both subsequent in-ROI contrast enhancement operations, and multitemporal image calibration. Following an iterative row-by-row search and crop routine, the lower leg emittance was segmented from the background using Otsu's histogram-based threshold selection method [9]. The masking method produced results similar to that in figure 1 , left.

\section{B. Intertemporal Image Calibration}

As detailed in protocol \#592-10 [5], we strove to consistently image recruits during weeks $1,4,7$ and 9 of basic training, as aligned with scheduled medical checkups and inoculation parades. Following segmentation, intertemporal images were calibrated by application of a global linear offset, as determined by histogram centroids, figure 1 


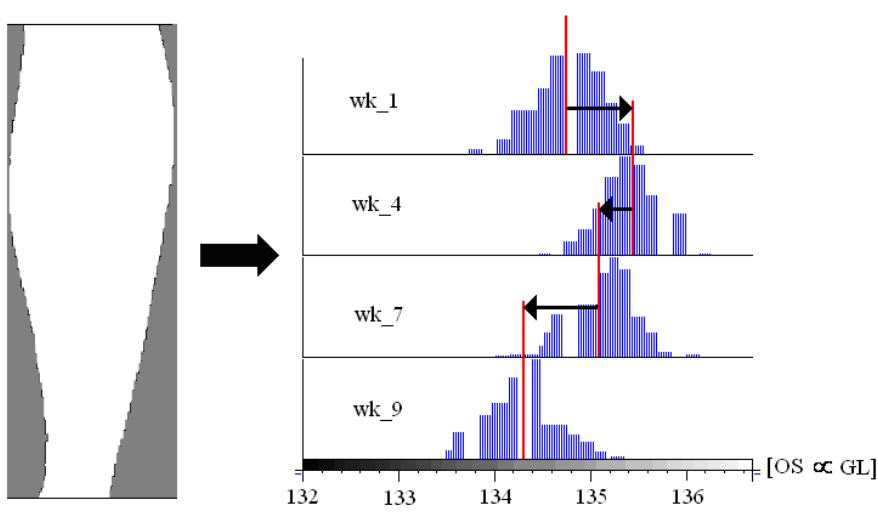

Fig. 1. Intertemporal image calibration offset taken from the centroids of the histogram representation of the segmented, emissively uniform, backgrounds of images taken from the same recruit during weeks 1, 4, 7, and 9 of their basic training, as detailed in [5].

right. As indicated by the uniform histogram widths in figure 1, this global linear offset application is valid.

\section{Enhancement}

During image acquisition, the TIRI system is set to detect emittance in a range closely encompassing ROIcharacteristic variation [4]. Following segmentation and subsequent masking of the background to a graylevel of zero; the width of each histogram in a multitemporal set is expressed as a ratio of the widest histogram in that set. This ratio is then used as a coefficient for a linear contrast stretch over all graylevels except 0 , which is occupied by the masked background. Following this selective normalization, each image was subject to contrast-limited adaptive histogram equalization. [10].

\section{PROCESSING}

As with all change detection applications, the preprocessed images (figure 2) must be searched for characteristic interest points. The authors identified SURF (speeded up robust features) which is a performant scaleand rotation-invariant interest point detector and descriptor [11]. SURF approximates or even outperforms previously proposed schemes with respect to repeatability, distinctiveness, and robustness, yet can be computed and compared much faster [11-13].

Having recently been found second most performant in a recent registration-specific evaluation of selected opensource implementations [13], OpenSURF [12] was selected for detection and characterization of interest points in this work. Moreover, [14] demonstrated OpenSURF's high performance in recognition of 3D non-planar objects based upon local grayscale, as applicable to the authors' ongoing work in registration of 2D TIRI's to 3D volumes rendered from thermometric MRI data sets $[4,6]$.
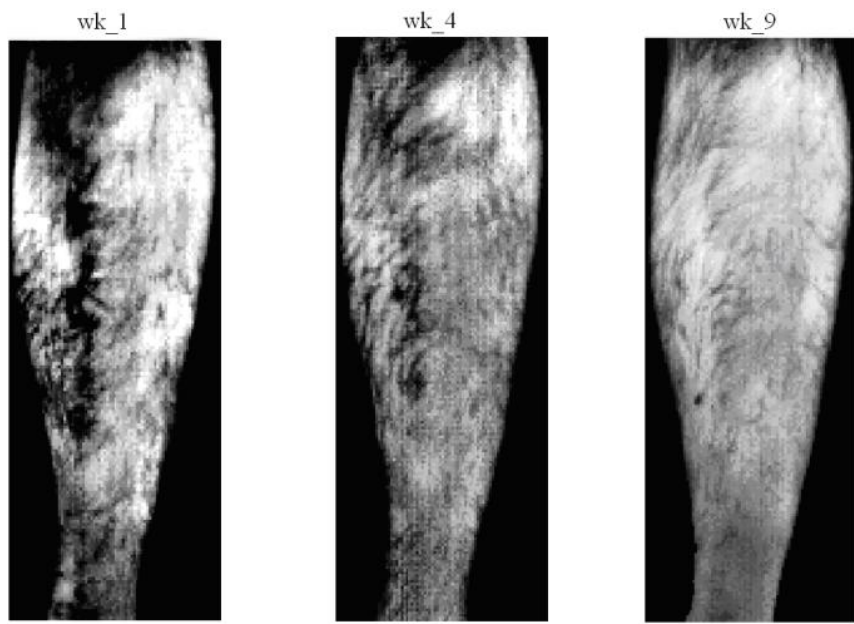

Fig. 2. Preprocessed multitemporal TIRI sequence from a symptomatic participant [5].

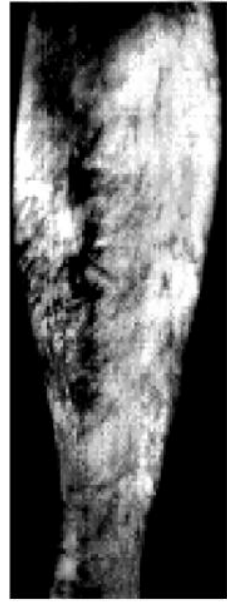

(a)

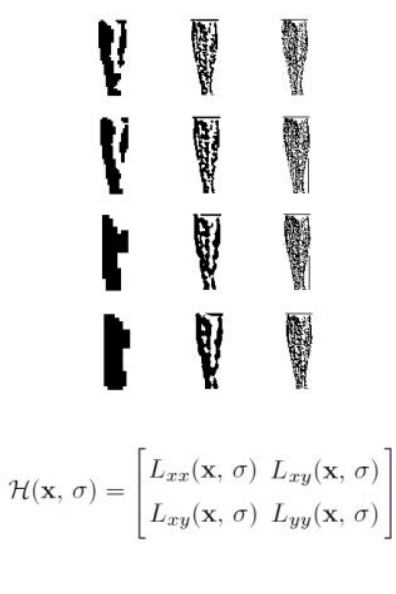

(b)

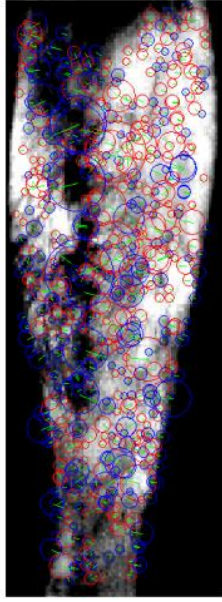

(c)
Fig. 3. Detection of interest points in a pre-processed TIRI (a) via the fast Hessian detector approximation (b) in OpenSURF. Detected interest points are plotted onto (a) in (c).

\section{A. Interest Point Identification}

The TIRI interest points were detected using OpenSURF's variant on the fast Hessian detector [12]. OpenSURF reduces computational expense in interest point detection via satisfactory approximation of the Hessian (figure 3b) discriminant via the Laplacian of Gaussians [12, 13]. Interest points detected by application of this method to figure $3 \mathrm{a}$, are plotted onto figure $3 \mathrm{c}$.

\section{B. Interest Point Characterization}

Once detected, interest points are characterized via local distribution, as classified by Haar wavelets, (figure 4). The descriptor entries of a sub-region represent the nature of the underlying intensity pattern. In figure 4 left, in the case of a homogeneous region, all values are relatively low. Middle: In presence of frequencies in $\mathrm{x}$ direction, the value of $|\mathrm{dx}|$ is high, but all others remain low. If the intensity is gradually 
increasing in the $\mathrm{x}$ direction, both values $\mathrm{dx}$ and $|\mathrm{dx}|$ are high

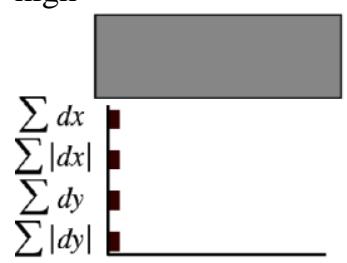

(a)

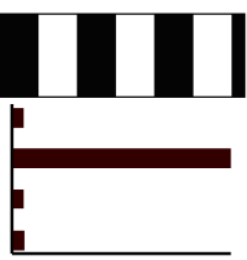

(b)

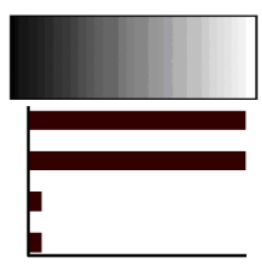

(c)
Fig, 4. SURF interest point descriptor based upon Haar wavelets [11].
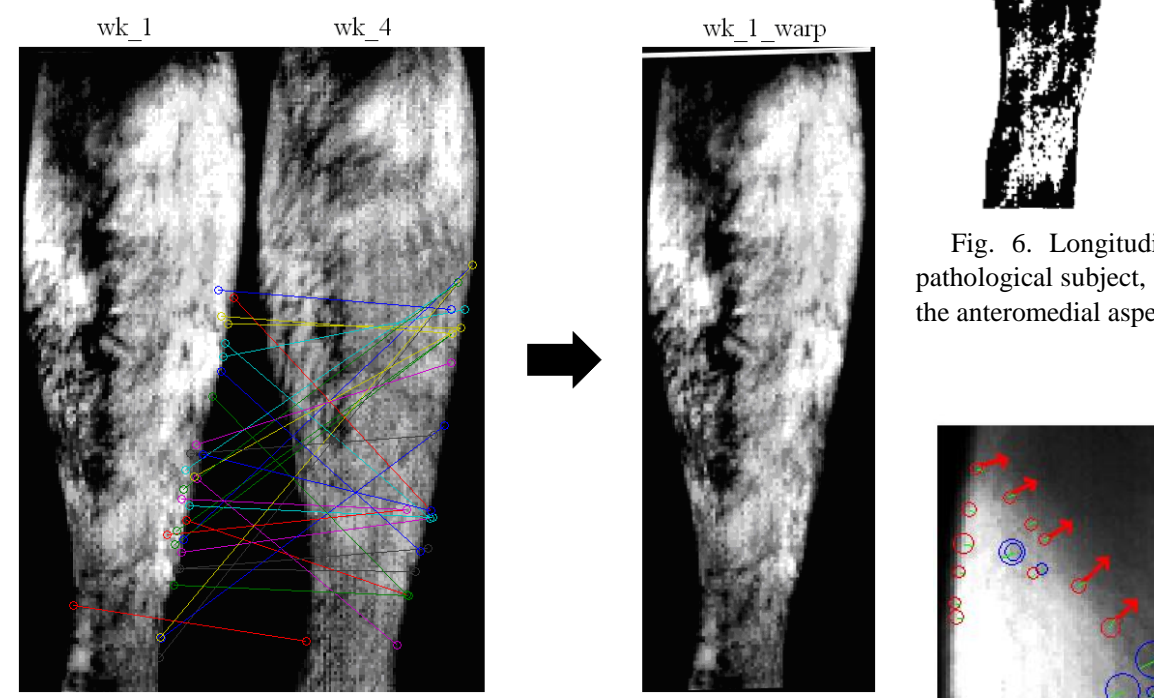

Fig. 5. OpenSURF-based generation of point correspondences and resultant warp of 'wk_1' image onto a 'wk_4' base image.

[11]. When applied to the TIRI's, the descriptor's performance benefitted greatly from previous segmentation and contrast enhancement. Once interest points in temporally consecutive images are characterized, those of most statistical similarity are assigned to each other in pairs, as visualized in figure 5, left.

\section{Difference Imaging}

Once interest points have been identified and characterized, they can be used as control points in registration, as in figure 5. As shown in figure 5 right 'wk_1_warp', the algorithm provides an excellent warp with minimal distortion to the original. Following such a highfidelity registration, it is meaningful to perform subtractive change detection, as in figure 6 below. Figure 6 shows consistency in the site of most emissive change over time; namely, the anteromedial aspect of the tibia.

This localization of change conforms to intuitive agreement with current understanding of osseous stress pathophysiology [15], and the thermophysiology of the underlying anatomy (figure 7). Specifically, the red arrows in figure 7 indicate the 'zip-up' optical flow of interest points at the interface of ROI's overlying the thermogenic muscle compartments and the relatively anemic / hemostatic
Fig. 6. Longitudinal difference imaging in a gradually progressing pathological subject, showing a consistent site of emissive change overlying

the anteromedial aspect of the tibia.
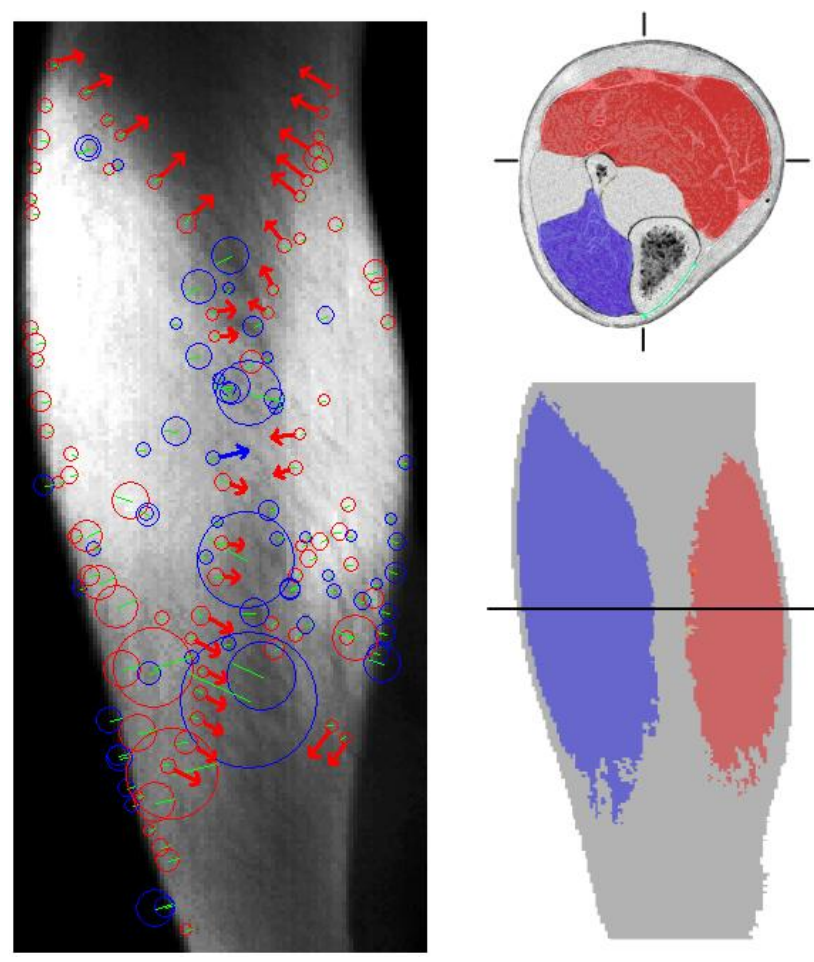

anteromedial pretibial.

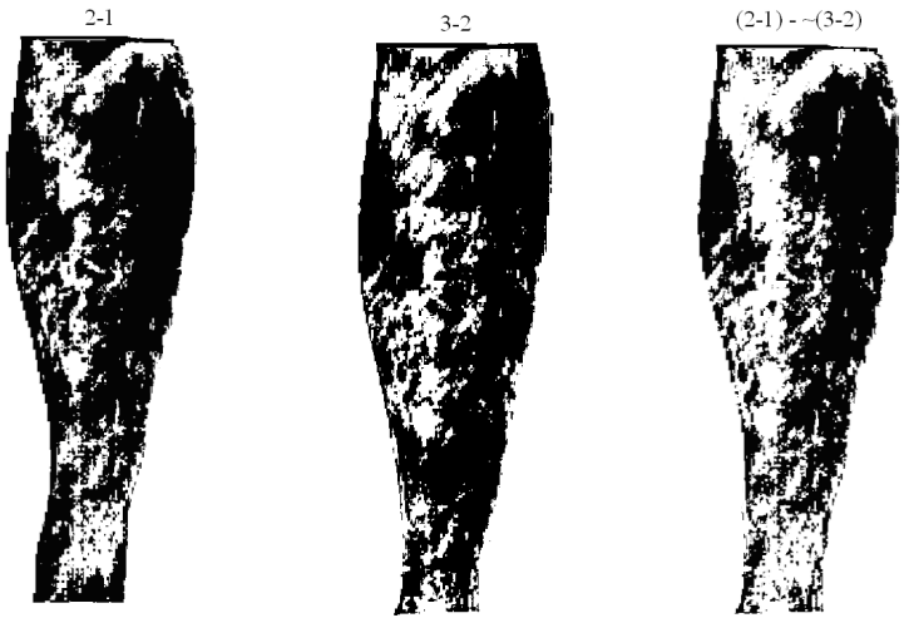

(1)

Fig. 7. Optical flow visualization of the progressive 'zipping up' of the emissive pre-muscle compartment regions over the pre-anteromedial tibial region with sustained stress; and correlation of detected TIR topography with underlying thermogenic anatomy.

In the authors' concomitant modeling and anatomic validation work (figure 8), initial results show promise for validation of representative etiological bioheat transfer models via registration of $2 \mathrm{D}$ TIRI's to $3 \mathrm{D}$ thermometric MRI volumes. 


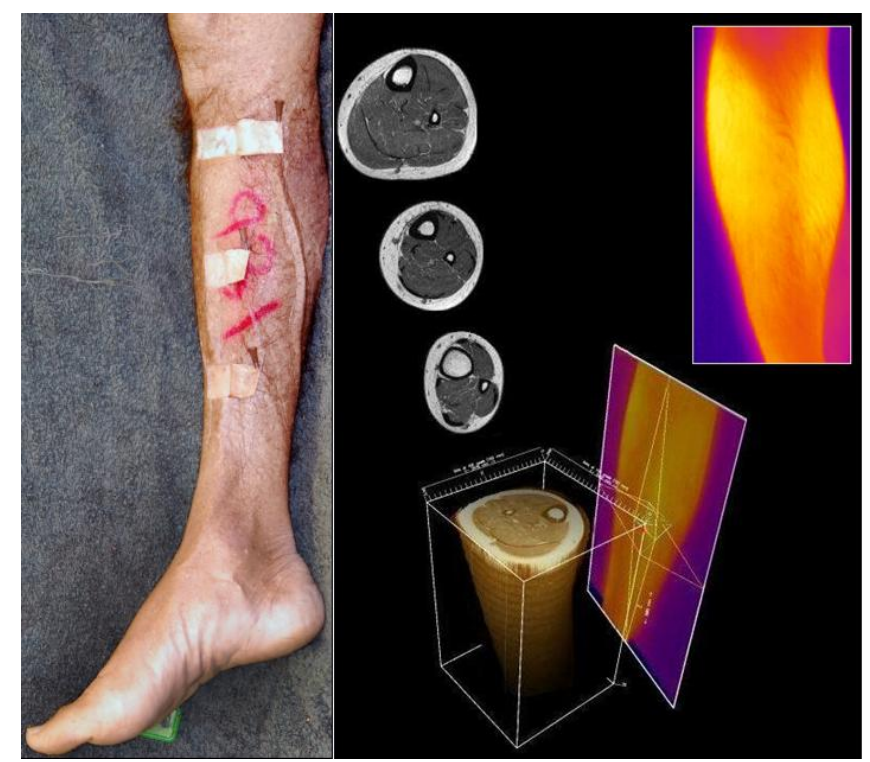

Fig. 8. Cadaveric excision of tissue volumes overlying the thermophysically distinct third of the anteromedial tibial diaphysis [16]; and conceptual visualization of high-fidelity 3D thermometric MRI volume generation and TIRI import.

\section{CONCLUSION}

A clinical image acquisition protocol suggested in [4] is validated, whereby the deliberate restriction of the scene to contain just the ROI and a known, uniformly emissive surface, allows both multitemporal calibration, and facilitates high- fidelity segmentation of the ROI from the scene. Modality- and application-appropriate algorithmic approaches to image processing and analysis are identified, justified, and demonstrated on clinically acquired longitudinal dataset. The SURF scale- and rotation-invariant interest point detector and descriptor algorithm has demonstrated high-fidelity registration in clinical TIRI data. In particular reference to osseous stress pathology, TIRI has shown potential for staging and perhaps even early detection in a representative sample of ten longitudinal 3 and 4 image sequences.

\section{ACKNOWLEDGMENT}

The authors would like to thank, the Australian Defence Force Human Research Ethics Committee, FLIR Systems Australia, Fremantle Hospital Imaging Services Division, and Dr. Andrew Squelch of the CSIRO's iVEC supercomputing facility.

\section{REFERENCES}

[1] L. B. Wolff, D. A. Socolinsky, and C. K. Eveland, "Face Recognition in the Thermal Infrared," in Computer Vision Beyond the Visible Spectrum, B. Bhanu and I. Pavlidis, Eds.: Springer-Verlag London Limited, 2005, pp. 167-191.
[2] E. Y. K. Ng and R. Acharya, "Remote Sensing Infrared Thermography," IEEE Engineering in Medicine and Biology, vol. 28, pp. 76-83, 2009.

[3] M. M. Khan, R. D. Ward, and M. Ingleby, "Classifying Pretended and Evoked Facial Expressions of Positive and Negative Affective States using Infrared Measurement of Skin Temperature," ACM Transactions on Applied Perception, vol. 6, pp. 1-22, 2009.

[4] D. Arthur and M. Khan, "Thermal Infrared Imaging: Toward Diagnostic Medical Capability," in proceedings of the 33rd Annual International Conference of the IEEE Engineering in Medicine and Biology Society, Boston MA, 2011, pp. 6146-6149.

[5] D. Arthur, M. Khan, and L. Barclay, "Thermographic Investigation of Osseous Stress Pathology," in proceedings of the 33rd Annual International Conference of the IEEE Engineering in Medicine and Biology Society, Boston MA, 2011, pp. 6250 - 6253.

[6] D. Arthur and M. Khan, "Toward Validation of TIRI as a Next Generation Deployable Health System," Journal of Military and Veterans' Health, vol. 3, pp. 1-9, 2011.

[7] P. Christensen, G. Mehall, S. Silverman, S. Anwar, G. Cannon, N. Gorelick, R. Kheen, T. Tourville, D. Bates, S. Ferry, T. Fortuna, J. Jeffryes, W. O'Donnell, R. Peralta, T. Wolverton, D. Blaney, R. Denise, J. Rademacher, R. Morris, and S. Squyres, "The Miniature Thermal Emission Spectrometer for the Mars Exploration Rovers," Journal of Geophysical Research vol. 108, pp. 1-21, 2003.

[8] S. Marchesi, "Advanced Pre-Processing and Change Detection Techniques for the Analysis of Multitemporal VHR Remote Sensing Images," Doctoral Thesis; University of Trento School of Information and Communication Technology, 2011.

[9] N. Otsu, "A Threshold Selection Method from Gray-Level Histograms," IEEE Transactions on Systems, Man, and Cybernetics, vol. 9, pp. 62-66, 1979.

[10] K. Zuiderveld, "Contrast Limited Adaptive Histograph Equalization," Graphic Gems IV, Academic Press Professional: San Diego, pp. 474485, 1994.

[11] H. Bay, A. T. Ess, T, and L. Van-Gool, "Speeded-Up Robust Features (SURF)," Journal Computer Vision and Image Understanding, vol. 110 , pp. 346-359, June 2008.

[12] C. Evans, "Notes on the OpenSURF Library," in The OpenSURF Computer Vision Library http://www.chrisevansdev.com/computervision-opensurf.html: www.chrisevansdevelopment.com 2011.

[13] D. Gossow, P. Decker, and D. Paulus, "An Evaluation of Open Source SURF Implementations," in Lecture Notes in Computer Science. vol. 6556, J. Ruiz-del-Solar, E. Chown, and P. Plöger, Eds.: Springer, 2011.

[14] P. Moreels and P. Perona, "Evaluation of features detectors and descriptors based on 3d objects," International Journal of Computer Vision, vol. 73, pp. 263-284, 2007.

[15] D. Pascoe, J. Mercer, and L. de Weerd, "Physiology of Thermal Signals," in Medical Infrared Imaging, N. Diakides and J. Bronzino, Eds. Boca Raton: CRC Press Taylor \& Francis Group, 2008, pp. 6.1 6.20 . 